\title{
Simple Method for Surface Selective Adsorption of Semiconductor Nanocrystals with Nanometric Resolution
}

\author{
O. Koslovsky, ${ }^{1}$ S. Yochelis, ${ }^{1}$ N. Livneh, ${ }^{1}$ M. G. Harats, ${ }^{2}$ R. Rapaport, ${ }^{1,2}$ and Y. Paltiel ${ }^{1}$ \\ ${ }^{1}$ Applied Physics Department and Center for Nanoscience and Nanotechnology, Hebrew University of Jerusalem, \\ 91904 Jerusalem, Israel \\ ${ }^{2}$ Racah Institute of Physics, Hebrew University of Jerusalem, 91904 Jerusalem, Israel
}

Correspondence should be addressed to O. Koslovsky, okjerusalem@gmail.com

Received 14 October 2012; Accepted 26 November 2012

Academic Editor: Chuanfei Guo

Copyright (C) 2012 O. Koslovsky et al. This is an open access article distributed under the Creative Commons Attribution License, which permits unrestricted use, distribution, and reproduction in any medium, provided the original work is properly cited.

\begin{abstract}
Self-assembly methods play a major role in many modern fabrication techniques for various nanotechnology applications. In this paper we demonstrate two alternatives for self-assembled patterning within the nanoscale resolution of optically active semiconductor nanocrystals. The first is substrate selective and uses any high resolution surface patterning to achieve localized self-assembly. The second method uses a surface with poly(methyl methacrylate) (PMMA) resist patterning adsorption of the nanocrystal with covalent bonds and liftoff.
\end{abstract}

\section{Introduction}

In recent years a major progress was achieved in fabrication and characterization techniques, down to nanometer scale resolution. This approach brings many opportunities and may open a way for developing new quantum engineered features as well as other important aspects. For example, in transport base devices, dense nanometric packaging of electrical devices increases the speed of operation, enhances the capacitance of a single chip, and reduces heating.

One promising approach is the use of self-assembled organic molecules as the major building blocks of these devices [1-3]. These devices usually relay on an assembly of $2 \mathrm{D}$ organic monolayers $[4,5]$. The combination of selfassembled organic molecules and semiconducting nanocrystals (NCs) is frequently employed as building blocks of such nanoscale electronics devices [6]. Optoelectronic properties of NCs can be controlled by their size, shape, composition, and doping which makes them attractive components in nanoelectronics devices [7]. In all these devices the distance between subsequent layers is nanometric, and the layers are self-ordered perpendicular to the substrate. However, the arrangement of the self-assembled layers in a controlled way is not fully developed yet and new methods that will help selective nanometric patterning are needed. Large efforts have been directed to achieve selective processes and several techniques were developed to answer the demand. In general the leading techniques can be divided into several classes: self-organization, nanoprinting, nanostamping, and photolithography patterning.

An example of nano printing is using surface patterning by microcontact chemistry, $[8,9]$ when a reactive "ink" molecule from a microstructured, elastomeric stamp is transferred onto surfaces modified with complementary reactive groups, leading to a chemical reaction in the area of contact. This process usually leads to micron-size resolution. By using solvent free microcontact printing with molded poly(dimethylsiloxane) (PDMS) as a nanostructure stamp template, Lee et al. showed deposition of patterned and unpatterned colloidal quantum dot thin films as the electroluminescent layers within hybrid organic-QD lightemitting devices. The result was a nanometric pattern of nanocrystal on a substrate. Self-organized systems can be based on templates of block copolymer films and include the fabrication of two-dimensionally organized nanoparticle assemblies on the patterning films as a simple and straight forward assembly mechanism [10]. The resolution in this case is nanometric but hard to control. 


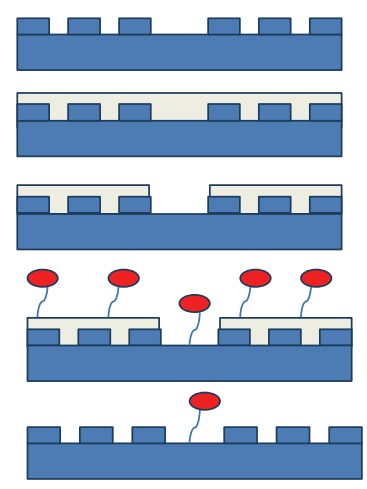

(a)

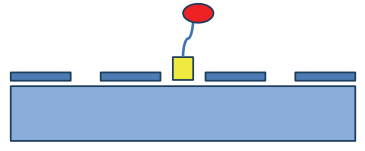

(b)
FIGURE 1: (a) Illustration of the selective adsorption process using resist and e-beam lithography to open a hole for molecules adsorption on a patterned metallic structure. (b) Illustration of selectively adsorbed nanoparticles by chemical specific recognition to gold surface.

New developed methods to the nano scale fabrication are based on the recently well-established lithography techniques. A significant work by Palma et al. demonstrated a selective self-assembly of biomolecules on nanopatterned surfaces together with minimizing nonspecific adsorption [11]. Another example is a study describing oleate-capped PbSe nanocrystals that were found to adhere preferentially to H-terminated Si surfaces over oxide and alkyl-terminated Si surfaces [12]. Scanning probe lithography was used to oxidize locally a dodecyl monolayer on the Si surface. Aqueous HF was then used to remove the oxide and expose $\mathrm{H}$-terminated $\mathrm{Si}$ areas. After exposure to a nanocrystals solution, local patterns of PbSe nanocrystals are created on the Si surface. The last patterning technique gives the highest resolution of selective adsorption; however, it requires the use of slow and expensive scanning probe microscopy writing and therefore is hard to adjust for industrial day to day use. Our approach uses two different methods to selectively adsorb nanocrystals with organic molecules. In both cases the patterned monolayers of the nanocrystals are realized using simple self-assembly methods. The high resolution surface patterning can be achieved on different substrate types due to molecules self-assembly. In our case we are utilizing e-beam lithography or evaporation method to prenanopattern the desired adsorbed area; however, any prepatterning options can be used to achieve selectivity based on the presented methods.

\section{Materials and Methods}

In the first method we cover a surface with poly(methyl methacrylate) (PMMA) 950 A2 resist. Using e-beam lithography we achieve nanometric resolution patterning as illustrated in Figure 1(a) for a patterned center of a round metallic lens surface. Next, self-assembly of organic molecules followed by nanoparticles adsorption is performed over the whole surface. With the removing of the resist the remaining nanocrystals are located only within the desired pattern

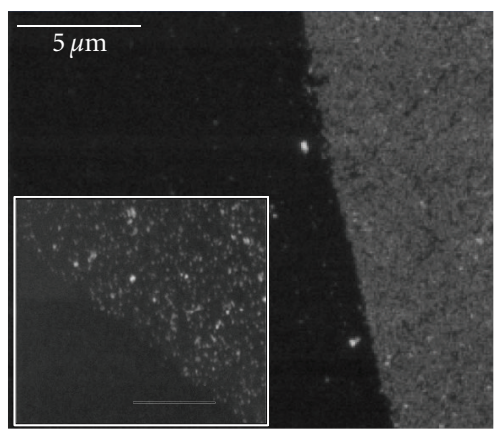

Figure 2: InAs/CdSe/ZnSe nanoparticles on top of a GaAs substrate. The sharp boarder is determined by the resolution of the e-beam lithography. The nanocrystals are chemically adsorbed to the surface using covalent bonds and therefore stay on the substrate after washing the sample in acetone and toluene. The inset shows the edge line at higher resolution.

(see illustration in Figure 1(a)). The covalent bonds holding the nanocrystals are strong enough to be compatible with repeatable process and therefore can be used to pattern layers.

In the second method we present a selective chemical recognition of different surfaces type by specific organic molecules groups. When evaporating gold nanopattern $(5 \mathrm{~nm}$ of $\mathrm{Ti}$ then $20 \mathrm{~nm} \mathrm{Au}$ ) onto oxidized surfaces the formed surfaces are chemically distinct. The adsorption of specific molecules like dithiol moieties takes place only onto the gold surfaces; therefore readsorption of nanoparticles to the tail end of the molecule results in specific regions with nanoparticles (see the illustration in Figure 1(b)).

The PMMA is usually patterned on a GaAs substrate. The substrate is washed with acetone and iso-propanol before oxygen-plasma asher cleaning. Then a PMMA is spin coated ( $5000 \mathrm{rpm}$ for $45 \mathrm{sec}$ ) on the cleaned substrate to have approximately $60 \mathrm{~nm}$ thick layer followed by prebaking at $180^{\circ} \mathrm{C}$ for $2 \mathrm{~min}$ (reflectometer measurement shows thickness of $78 \mathrm{~nm}$ ). The desired pattern is achieved by opening lines in the PMMA with e-beam lithography. The sample is then developed in $5^{\circ} \mathrm{C}$ methyl isobutyl ketone (MIBK): IPA for $120 \mathrm{sec}$. The PMMA liftoff after NCs adsorption is performed in a hot acetone for $2 \mathrm{~min}$ then sonicated in heated acetone for $1 \mathrm{~min}$ and finally washed with isopropanol (IPA) and dried with $\mathrm{N}_{2}$.

Both of the above procedures use self-assembly organization on the $\mathrm{Au}, \mathrm{GaAs}$, or $\mathrm{Si}$ substrates. All the substrates are rinsed with acetone then ethanol and treated with 50\% plasma asher power (Diener PICO UHP $40 \mathrm{kHz} / 200 \mathrm{~W}$ ) for $10 \mathrm{~min}$, prior to molecule adsorption. When using GaAs the substrate is also etched with $2 \% \mathrm{HF}$ for $5 \mathrm{sec}$ followed by $25 \%$ $\mathrm{NH}_{3} \mathrm{OH}$ for $30 \mathrm{sec}$ prior to the molecule self-assembly. It is then incubated in ethanol solution for 20 minutes, followed by incubation in $1 \mathrm{mM} 1,9$-nonanedithiol in anhydrous ethanol solution overnight and finally the substrate is dipped in InAs/CdSe/ZnSe nanocrystals suspended in Toluene for 4 hours. The sample is then washed in Toluene for several times. 


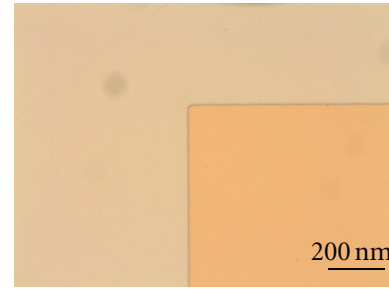

(a)

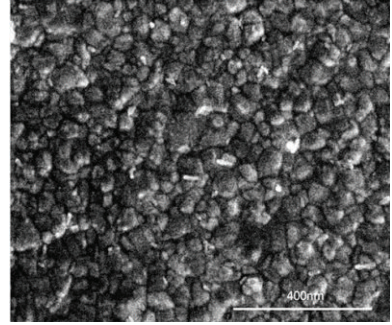

(b)

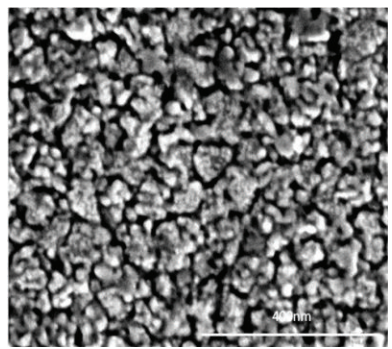

(d)

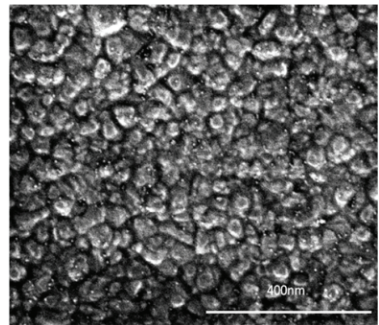

(c)

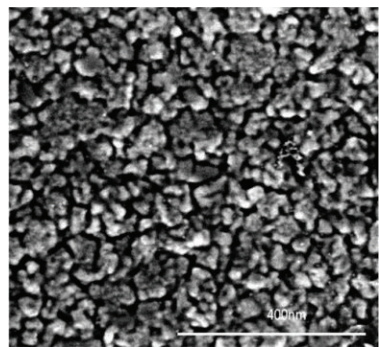

(e)

FIguRE 3: Specific adsorption demonstrated on an array of gold squares on an aluminum surface. The sample is shown in (a). The smaller square is the gold surface and at the background is the aluminum substrate. In (b and d) the gold and aluminum substrates, respectively, are shown with molecules adsorption only. In (b) It is possible to observe the gold grains but the surface is clean from NCs. (c) shows the gold area of the sample after dipping the sample in the NCs solution. The surface is covered with NCs at a density of about $7 \times 10^{10} \mathrm{NCs} / \mathrm{cm}^{2}$. By comparing these results to the adsorption of NCs on the $\mathrm{Al}_{2} \mathrm{O}_{3}$ surface (d) with molecules only and (e) after dipping in NCs solution, we can see the selectivity.

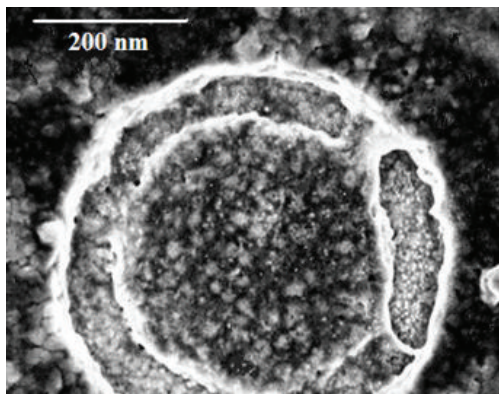

Figure 4: High resolution SEM images of aluminum and gold lens structure. Nanometric resolution of specific adsorption is demonstrated on the $300 \mathrm{~nm}$ diameter gold surface in the center of the aluminum sample. The gold center part is brighter than the aluminum part. NCs are seen only on the gold.

The InAs/CdSe/ZnSe nanocrystals (from Prof. Uri Banin's group) were synthesized in tri-octyl phosphine using $\mathrm{InCl}_{3}$ and $\mathrm{TMS}_{3}$ As as precursors according to the procedure described elsewhere [13] and underwent subsequently a size-selection process. For the purposes of the experiment we have used InAs NCs $6.5 \mathrm{~nm}$ in diameter. For the shell growth an ionic layer adsorption and reaction process has been used. The shell consisted of 1 monolayer (ML) of CdSe, followed by $4 \mathrm{MLs}$ of $\mathrm{ZnSe}$. As a result, the emission efficiency from the NCs at $0.96 \mathrm{eV}$ has increased by more than 2 orders of magnitude.

\section{Main Results}

Specific adsorption using PMMA resist patterning on a GaAs substrate is presented in Figure 2. In this figure a clear line is seen between the area of dense InAs/CdSe/ZnSe NCs adsorption $\left(10^{12} \mathrm{NCs} / \mathrm{cm}^{2}\right)$ on the right where the resist was developed and the places where the liftoff occurred. This high selectivity line is presented at larger scale at the inset.

In this method the adsorption is performed all over the sample and after removing the resist, in our case a PMMA, the desired nanocrystal pattern emerges. It is then possible to have very high resolution patterns depending on the e-beam lithography resolution limits. This procedure is not limited to the surface type and also it is possible to repeat the process several times on the same sample in order to get multilayers.

Another option we present to selectively pattern selfassembled layers is demonstrated by molecular specific chemical recognition. We show before a clear border between nanoparticles adsorbed on one surface and a different surface that shows no adsorption. In Figure 3(a) we show an array of gold squares on an aluminum surface. The figure presents a selective adsorption of InAs/CdSe/ZnSe nanocrystals to the gold parts of the sample. The selectivity here occurs due to a specific chemical recognition between thiol ( $\mathrm{SH}$ ) head group to a gold surface but not to the oxide surfaces of the sample. In Figures 3(b), 3(c), 3(d), and 3(e) the specific adsorption is demonstrated. Here again a thiol group was adsorbed to a gold surface but not to aluminum. In this process, in order to prevent NCs to physically adsorb to the aluminum surface, 


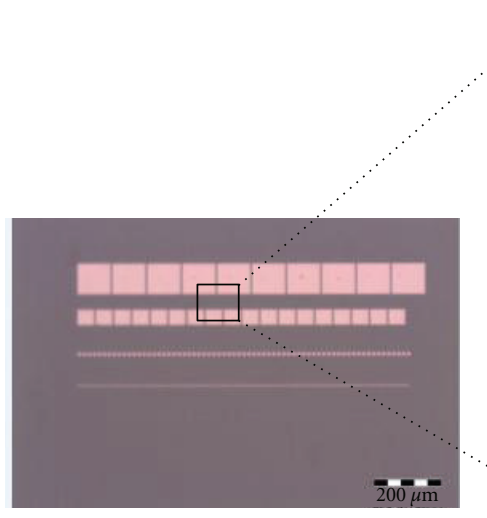

(a)

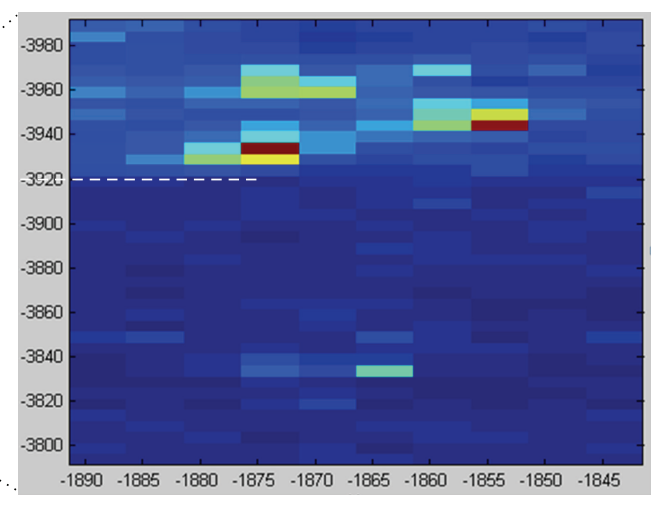

(b)

Figure 5: On the left, the patterned gold on Si sample, on the right optical mapping of the emission from the NCs. The dashed line shows the gold (top)/Si (bottom) border.

we adsorbed also octadecyl trichlorosilane (OTS) molecules that attach to the aluminum surface. This monolayer does not have a tail group to bind to the NCs. Figure 3(b) represents gold substrate with 1,9-nonane dithiol molecules adsorbed only. It is possible to observe the gold grains but the surface is clean from NCs. On the contrary, in Figure 3(c) we show the same sample after dipping it in the NCs solution where we observe the surface which is covered with NCs. The NCs density on the surface is about $7 \times 10^{10} \mathrm{NCs} / \mathrm{cm}^{2}$. Comparing these results to the adsorption of NCs on the $\mathrm{Al}_{2} \mathrm{O}_{3}$ surface in Figures $3(\mathrm{~d})$ and $3(\mathrm{e})$ it is possible to see that the surface is almost clean from NCs also after dipping (Figure 3(e)) in the NCs solution. XPS measurements on the gold and aluminum surfaces confirmed the selectivity of the adsorption: on the gold surface the signal from the NCs was much higher. The presented chemical specific recognition of molecules to a certain surface together with photoresist patterning opens the opportunity of creating complicated nanoresolution patterns.

To realize selective adsorption to structures with nanometric resolution, we prepared a $300 \mathrm{~nm}$ diameter gold surface in the center of concentric aluminum ring. This is important, for example, for applications where the emission of photons from the NCs should be directional [14-16]. In this case it is important to adsorb the NCs at the center of the lens only. By fabricating a nanometric gold surface at the center of the lens one can use selective adsorption to guarantee that the NCs will be located at the center of the lens only. Figure 4(a) is showing high resolution SEM images of the concentric gold circle surrounded by such an aluminum circle. The NCs are adsorbed only onto the gold area and not on the surrounding aluminum part.

Finally in order to check that the NCs adsorb to gold selectively, with organic linkers that are long enough to decrease the nonradiative coupling to the metal substrate and prevent significant optical quenching, we have performed spatially resolved optical measurements with a high resolution confocal detection setup. The InAs/CdSe/ZnSe NCs were excited by a $780 \mathrm{~nm}$ laser and their emission was around $1200 \mathrm{~nm}$. The dots were highly concentrated on the gold part with density higher than $10^{11} \mathrm{dot} / \mathrm{cm}^{2}$. We have achieved the same selectivity using both micrometric and nanometric patterns. Down to the $100 \mathrm{~nm}$ size the selectivity is not size dependent, and therefore the same procedure can be used with any high resolution nanopatterning. Figure 5(b) displays optical mapping of the NCs emission of the sample that is presented in Figure 5(a). The dashed white line presents the gold/Si border. The top part of the sample map is the gold substrate where a clear emission from the NCs is observed, while on bottom of the Si part almost no NCs are recognized. The exact patterning border seems to be smaller than $40 \mathrm{~nm}$ and is related to our fabrication quality.

\section{Summary}

In summary we demonstrate self-assembled patterning with nanoscale resolution using two methods: e-beam lithography and selective chemical recognition of surfaces. The prenanopatterning employs e-beam lithography or evaporation in our case; however, any prepatterning process could be used in order to achieve the needed selectivity. The high resolution patterning achieved utilizing both known methods as ebeam lithography and evaporation, and self-assembly of organic molecules. The processes are simple and compatible with many substrates. Moreover, it is possible to repeat the processes several times to achieve multilayers on the selected surfaces.

\section{References}

[1] N. J. Tao, "Electron transport in molecular junctions," Nature Nanotechnology, vol. 1, no. 3, pp. 173-181, 2006.

[2] P. E. Burrows, S. R. Forrest, and M. E. Thompson, "Prospects and applications for organic light-emitting devices," Current Opinion in Solid State and Materials Science, vol. 2, no. 2, pp. 236-243, 1997.

[3] H. E. Katz and Z. Bao, "The physical chemistry of organic field-effect transistors," Journal of Physical Chemistry B, vol. 104, no. 4, pp. 671-678, 2000.

[4] H. B. Akkerman, P. W. M. Blom, D. M. De Leeuw, and B. De Boer, "Towards molecular electronics with large-area molecular junctions," Nature, vol. 441, no. 1, pp. 69-72, 2006. 
[5] O. Seitz, A. Vilan, H. Cohen et al., "Doping molecular monolayers: effects on electrical transport through alkyl chains on silicon," Advanced Functional Materials, vol. 18, no. 14, pp. 2102-2113, 2008.

[6] Y. Paltiel, A. Aharoni, U. Banin, O. Neuman, and R. Naaman, "Self-assembling of InAs nanocrystals on GaAs: the effect of electronic coupling and embedded gold nanoparticles on the photoluminescence," Applied Physics Letters, vol. 89, no. 3, Article ID 033108, 3 pages, 2006.

[7] X. Peng, L. Manna, W. Yang et al., "Shape control of CdSe nanocrystals," Nature, vol. 404, no. 6773, pp. 59-61, 2000.

[8] C. Wendeln and B. J. Ravoo, "Surface patterning by microcontact chemistry," Langmuir, vol. 28, no. 13, pp. 5527-5538, 2012.

[9] A. Lee, P. O. Anikeeva, S. A. Coe-Sullivan, J. S. Steckel, M. G. Bawendi, and V. Bulović, "Contact printing of quantum dot light-emitting devices," Nano Letters, vol. 8, no. 12, pp. 45134517, 2008.

[10] M. J. Pavan and R. Shenhar, "Two-dimensional nanoparticle organization using block copolymer thin films as templates," Journal of Materials Chemistry, vol. 21, no. 7, pp. 2028-2040, 2011.

[11] M. Palma, J. J. Abramson, A. A. Gorodetsky et al., "Selective biomolecular nanoarrays for parallel single-molecule investigations," Journal of the American Chemical Society, vol. 133, no. 20, pp. 7656-7659, 2011.

[12] G. P.-A. Andras, H. Qiao, J. Shan et al., "Site-selective optical coupling of pbse nanocrystals to si-based photonic crystal microcavities," Nano Letters, vol. 9, no. 8, pp. 2849-2854, 2009.

[13] A. A. Guzelian, U. Banin, A. V. Kadavanich, X. Peng, and A. P. Alivisatos, "Colloidal chemical synthesis and characterization of InAs nanocrystal quantum dots," Applied Physics Letters, vol. 69, no. 10, pp. 1432-1434, 1996.

[14] H. J. Lezec, A. Degiron, E. Devaux et al., "Beaming light from a subwavelength aperture," Science, vol. 297, no. 5582, pp. 820 822, 2002.

[15] A. G. Curto, G. Volpe, T. H. Taminiau, M. P. Kreuzer, R. Quidant, and N. F. Van Hulst, "Unidirectional emission of a quantum dot coupled to a nanoantenna," Science, vol. 329, no. 5994, pp. 930-933, 2010.

[16] N. Livneh, A. Strauss, I. Schwarz et al., "Highly directional emission and photon beaming from nanocrystal quantum dots embedded in metallic nanoslit arrays," Nano Letters, vol. 11, no. 4, pp. 1630-1635, 2011. 

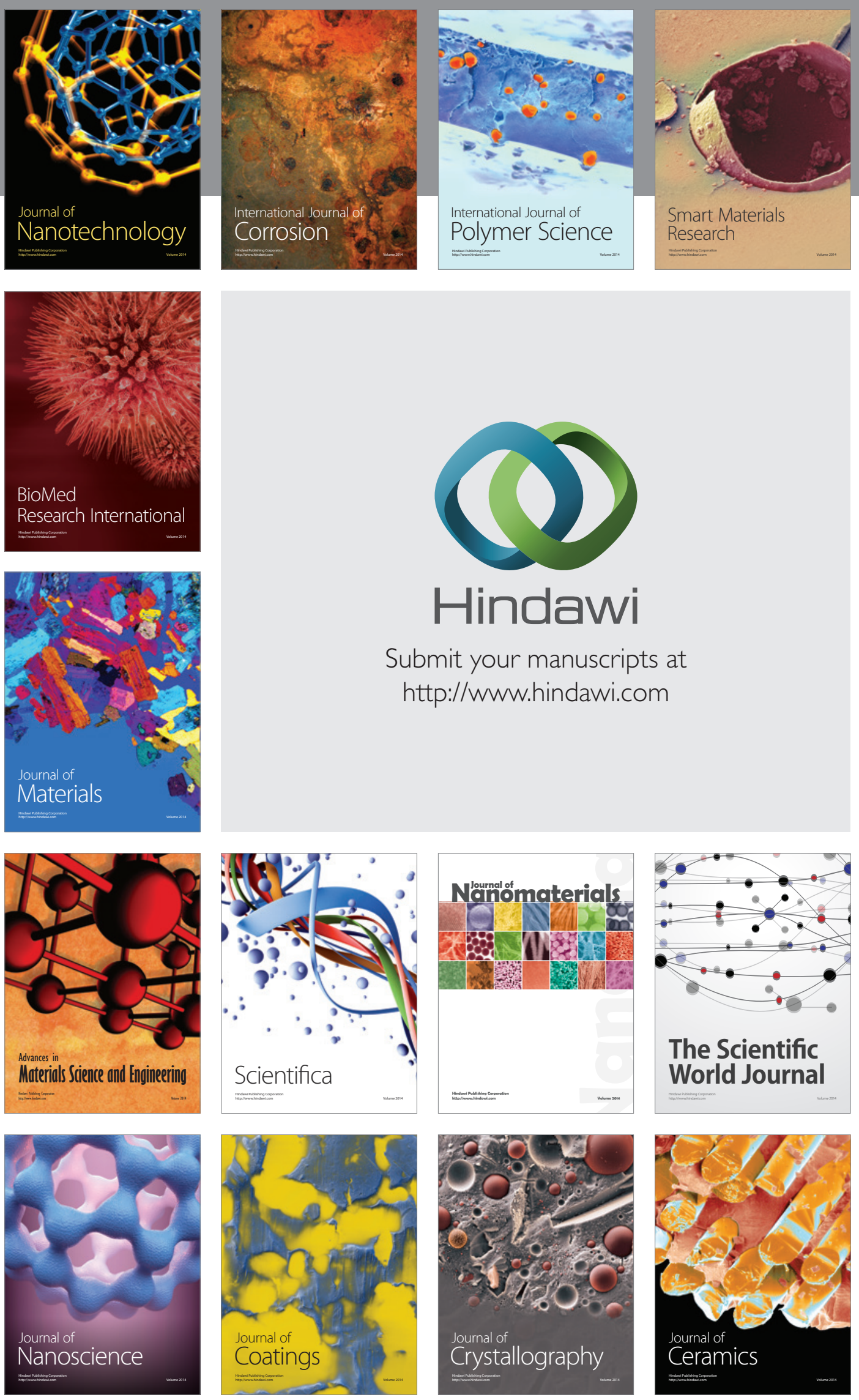

The Scientific World Journal

Submit your manuscripts at

http://www.hindawi.com

\section{World Journal}

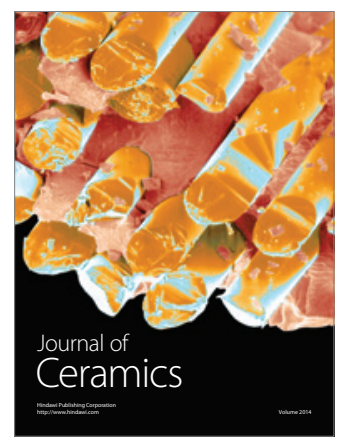

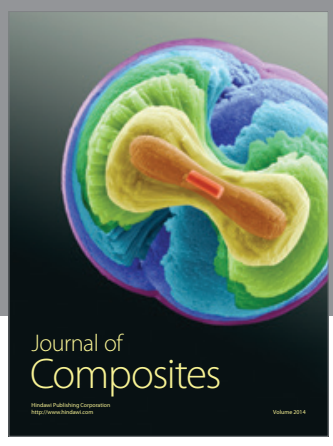
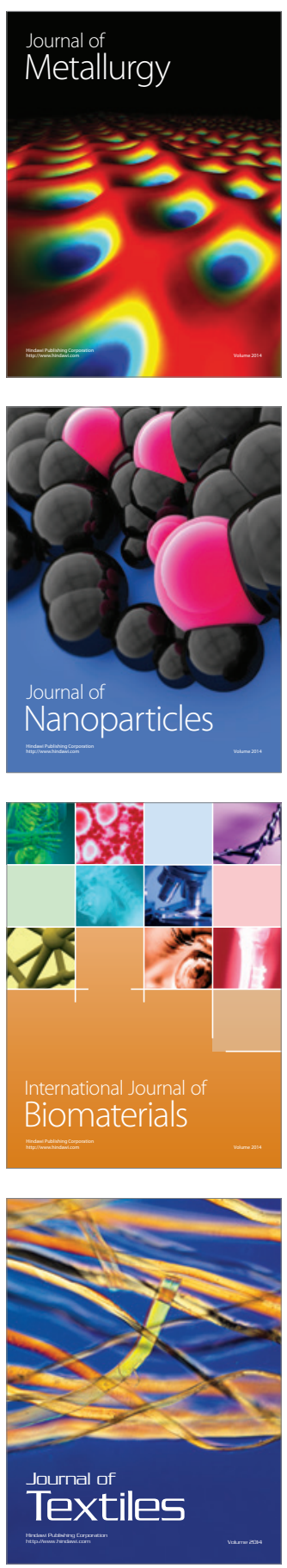